\title{
Improving Electromagnetic Interference Shielding Effectiveness using Copper Reinforced Sustainable Foam
}

\author{
Manshu Gupta \\ ${ }^{1}$ Department of Mechanical Engineering, \\ School of Mechanical and Building Sciences \\ VIT University Chennai Campus, \\ Chennai- 600127, Tamil Nadu, India

\section{Ashutosh Dubey ${ }^{1}$} \\ ${ }^{1}$ Department of Mechanical Engineering, \\ School of Mechanical and Building Sciences \\ VIT University Chennai Campus, \\ Chennai-600127, Tamil Nadu, India.
}

\author{
Timir Patel ${ }^{1}$ \\ ${ }^{1}$ Department of Mechanical Engineering, \\ School of Mechanical and Building Sciences \\ VIT University Chennai Campus, \\ Chennai- 600127, Tamil Nadu, India \\ Jeyanthi Subramanian ${ }^{2}$ \\ ${ }^{2}$ Associate Professor, \\ Department of Mechanical Engineering, \\ School of Mechanical and Building Sciences, \\ VIT University Chennai Campus Chennai-600127, Tamil \\ Nadu, India.
}

\begin{abstract}
Organic foams with different proportions of copper powder are fabricated using a general mixing process. The physical and chemical properties and the electromagnetic shielding effectiveness of these foams are investigated. The results indicate the presence of different organic functional groups. Copper foams have Electromagnetic interference shielding(EMI) effectiveness of around $10 \mathrm{~dB}$ within the frequency of 8-12 GHz. The EMI shielding effectiveness does not change with increasing the proportion of copper powder. On the other hand, the organic foams with a rectangular $2 \mathrm{~mm}$ fine steel mesh has a good EMI shielding effectiveness of around $30 \mathrm{~dB}$ within the frequency range of $8-12 \mathrm{GHz}$
\end{abstract}

Keywords-Organic Foam, Copper Powder, Electromagnetic Interference (EMI)Shielding, Frequency, Fine steel mesh

\section{INTRODUCTION}

The increasing complexity of electronic devices or systems in the form of higher packing density for quick response has resulted in electromagnetic interference. ${ }^{[1]}$ The development in the use of electromagnetic devices over a wide range of mechanical, business and consumer parts has made another type of contamination known as noise or radio frequency interference (RFI) or electromagnetic interference (EMI).EMI consists of many unwanted radiated signals which can cause unacceptable degradation of system or equipment performance. ${ }^{[2]}$ In a specific environment, an appropriate shielding material should be selected in order to obtain a good effect. So it is necessary to research the performance features of the potential EMI shielding materials. ${ }^{[3-6]}$

In a particular situation, a fitting protecting material ought to be chosen so as to acquire a decent impact. So, it is important to look into the execution highlights of the potential EMI protecting materials. In this way, there is a necessary requirement for the effective protecting of equipment from its unfavorable impacts.

The organic foam synthesized is to be tested for shielding of electromagnetic interference. The merits of using an organic foam is that it is lightweight, flexible and eco-friendly. Five test samples are synthesized one of which is a flexible organic foam without additives, one is a rigid foam without additives, three of them are flexible foams with varying proportions of copper powder by weight, one is a flexible foam with a $2 \mathrm{~mm}$ steel fine mesh in the midplane and one is a rigid foam with a $2 \mathrm{~mm}$ steel fine mesh in the midplane. Various techniques are used to obtain the properties of the samples. A shielding effectiveness test setup is used for testing the EMI shielding capability of the material

\section{EXPERIMENTL PROCEDURE}

\section{A. Fabrication of copper Foam and Meshed Foam}

Two different types of samples have been prepared namely the flexible and rigid foams. The flexible foams had a common mixture of isocyanides and polyol in the ratio of $2: 3$. First of all, an organic foam with no additives was prepared by adding the two compounds in a cuboidal mould with one face open and letting the reaction take place. The reaction is exothermic and carbon dioxide gas is released. The mixture is allowed to expand and is then removed from the mould and is prepared according to the required dimensions. Now, three different samples were prepared with different weight by weight percentages of copper powder, i.e., $3 \%, 5 \%$ and $10 \%$, in a similar manner as more than $10 \%$ of copper powder reaction were not able to complete and mixture was collapsed. The three compounds including the copper powder are added in the mould. Another flexible foam sample was prepared by placing a sheet of $2 \mathrm{~mm}$ fine steel mesh in the mid-plane of the mould and adding the organic compounds, isocyanides and polyol and letting the reaction take place. The rigid foams were prepared using the two organic compounds, isocyanides and castor oil, in the ratio $2: 3$. The sample is prepared using the same process used for preparing the flexible foam and the same mould. One sample using the steel mesh is prepared using the above described process. The sample for 
preparation of rigid foam containing the copper powder were not able to complete the reactions and collapsed.

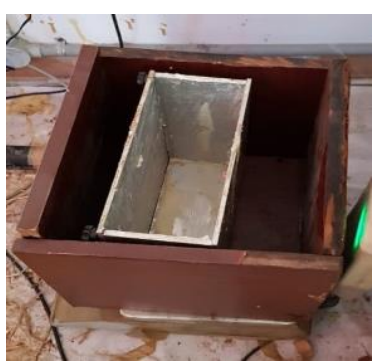

Fig. 1. Specimen Mould

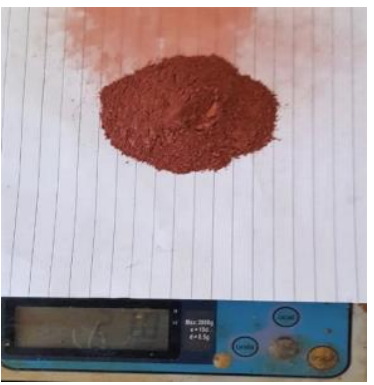

Fig. 3. Measurement Apparatus

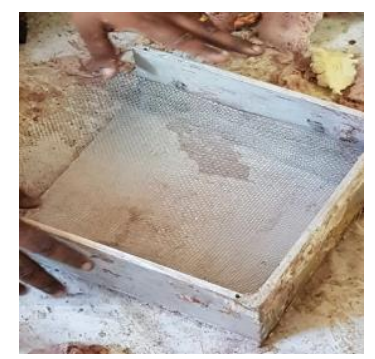

Fig. 2. Al tray with wax on surface

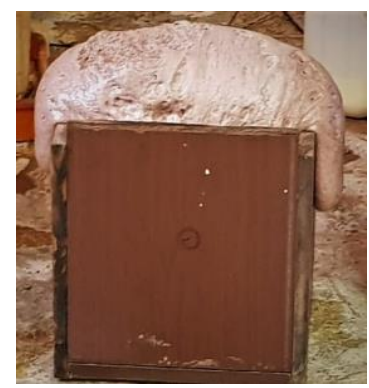

Fig. 4. Final Product

\section{B. EMI shielding test Setup}

The electromagnetic interference (EMI) shielding effectiveness is measured by using an experimental setup consisting of a transmitter transmitting a radiation of $8 \mathrm{GHz}$ - $12 \mathrm{GHz}$ and a receiver. An analyzer is used to generate a plot of the Shielding Effectiveness (SE) of the sample being tested. In this setup, the transmitter and the receiver are placed $30 \mathrm{~cm}$ apart and the sample is placed in between as shown in the photograph. The plot is obtained on the analyzer for the frequency range of $8 \mathrm{GHz}-12 \mathrm{GHz}$.

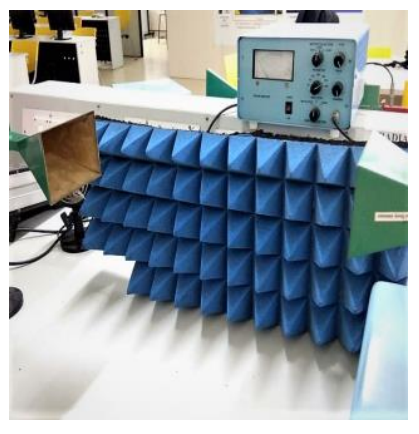

Fig. 5. Setup With Absorber

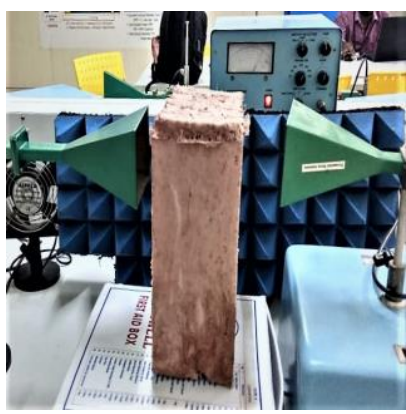

Fig. 7. Setup for Copper sample

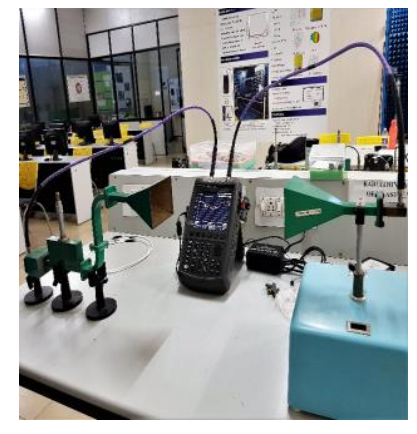

Fig. 6. Setup with analyser

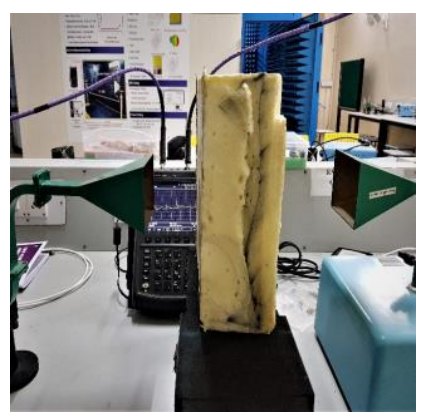

Fig. 8. Setup for meshed sample

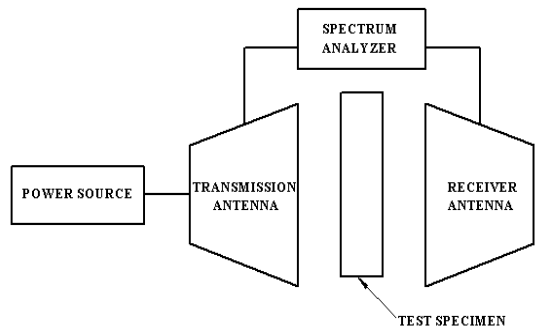

Fig. 9. Schematic Diagram of the Shielding Effectiveness Test

\section{RESULT AND DISCUSSION}

\section{A. FTIR Test}

According to the plots obtained from the Fourier Transformation Infrared (FTIR) Spectrum, we have verified the functional groups present in the different foams. The plots obtained are shown below.

- Flexible Foam: In the above graph, we can observe the broadening of the peaks in the range near $3500 \mathrm{~cm}^{-1}$ which confirms the presence of $-\mathrm{OH}$ functional group. The stretching of the peaks near the range of $3000 \mathrm{~cm}^{-1}$ confirms the presence of $-\mathrm{C}$ $\mathrm{H}$ groups. The absorption in the range of 2165 $2110 \mathrm{~cm}^{-1}$ shows the presence of cyanide group.

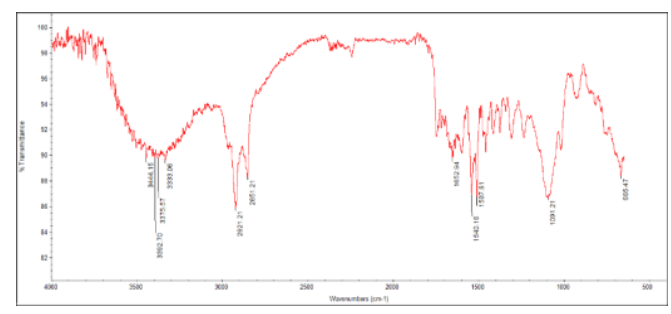

Fig. 10. Flexible Organic Foam

- Rigid Foam: The broadening of the peaks in the range near $3500 \mathrm{~cm}^{-1}$ which confirms the presence of $-\mathrm{OH}$ functional group. The stretching in the range of $1750-1715 \mathrm{~cm}^{-1}$ confirms the presence of $\mathrm{C}=\mathrm{O}$ functional group and the stretching in the range of $1300-1000 \mathrm{ccm}^{-1}$ confirms the presence of $\mathrm{C}-\mathrm{O}$ functional group. Thus, the presence of ester functional group is confirmed. Thus, the presence of ricin oleic acid which is the main component of castor oil is confirmed. The absorption in the range of $2165-2110 \mathrm{~cm}^{-1}$ shows the presence of cyanide group. The expansion in the range of 1680-1640 $\mathrm{cm}^{-1}$.

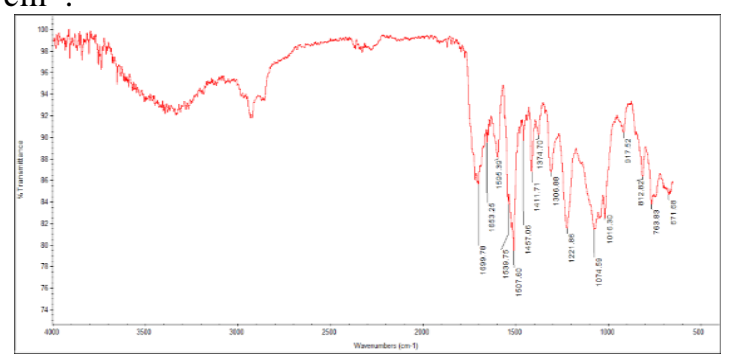

Fig. 11. Rigid Organic Foam 


\section{B. EMI Shielding Performance Test}

According to the graphs obtained from the EMI shielding effectiveness experiment, the flexible foam with steel mesh was able to provide a shielding effectiveness (SE) of $27 \mathrm{~dB}$ to $31 \mathrm{~dB}$ in the range of $8 \mathrm{GHz}-12 \mathrm{GHz}$. The rigid foam with steel mesh was able to provide a SE of 25 $\mathrm{dB}$ to $30 \mathrm{~dB}$ in the range of $8 \mathrm{GHz}-12 \mathrm{GHz}$. The $\mathrm{SE}$ of the foam with no additives and the ones with the copper powder additive was comparable as that of free air which was nearly negligible with the values having maxima around $-14 \mathrm{db}$ to $-15 \mathrm{db}$ and minima around $-9 \mathrm{db}$ to $-10 \mathrm{db}$.

\section{Frequency v/s Return Loss}

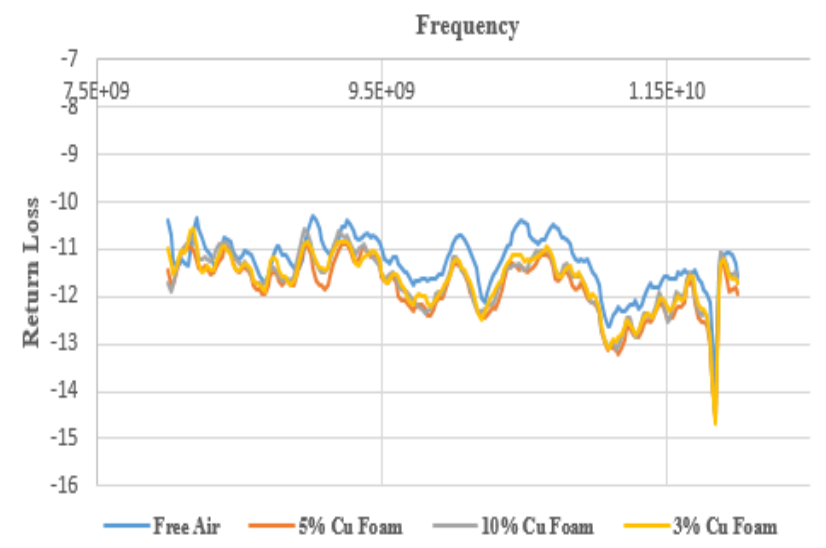

Shielding Effectiveness(SE) of different proportions of copper in the Organic Foam

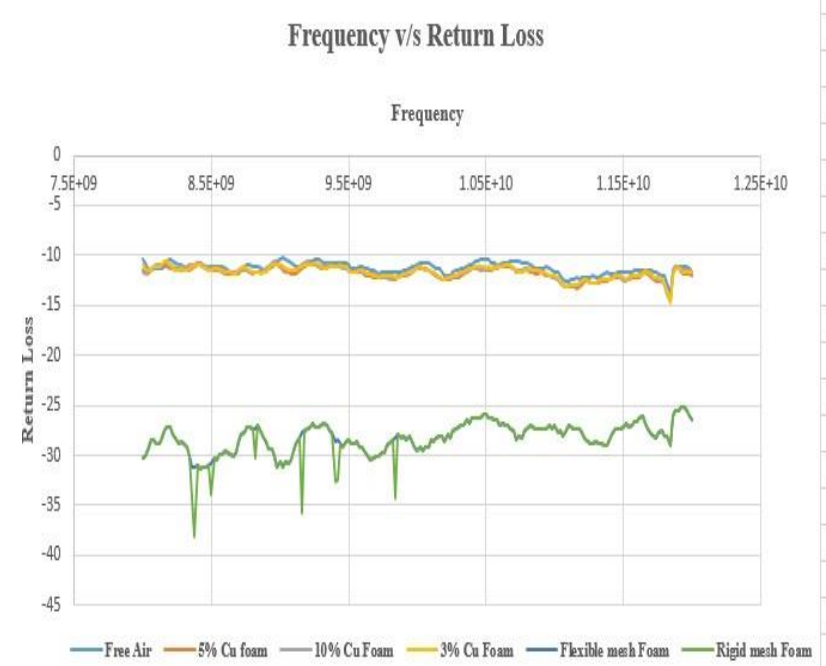

Fig 13. Comparison of SE of different proportions of copper with Steel Mesh in the Organic Foam

Thus, it can be implied that the organic foam with no additive has a negligible shielding effect in the range of $-10 \mathrm{db}$ to a maximum of $-15 \mathrm{db}$ and even the addition of copper powder to the organic foam did not enhance the shielding effectiveness of the material. On the other hand, the foam consisting of the steel mesh has a significant effect on the shielding capability of the foam.

\section{CONCLUSIONS}

- Copper powder reinforced forms with three different proportions of copper were prepared. Along with these, two more samples with a fine steel mesh were also prepared. The flexible foams primarily contain polyol and isocyanides and the rigid forms contain castor oil and isocyanides.

- The physical and chemical properties of the samples are characterized and the shielding effectiveness is characterized using EMI performance test setup.

- The shielding effectiveness of the rigid and flexible foams with steel mesh were observed to be around 25-30 $\mathrm{dB}$ and the copper foams show little shielding against the waves.

- Thus, we can conclude that in the frequency range of $8 \mathrm{GHz}$ to $12 \mathrm{GHz}$, the flexible and rigid organic foams with steel mesh can be actively used to provide effective shielding for satellite communications body parts covering, radio navigation and location, terrestrial data transfer and radio operations.

- Also, the samples that do not provide shielding in the frequency range of $8 \mathrm{GHz}$ to $12 \mathrm{GHz}$ can be utilized for transmitting purposes without interruptions and providing an external covering in the areas of satellite communications, terrestrial data transfer and radio operations by providing a cover to the equipment's and between the devices.

\section{ACKNOWLEDGEMENT}

We are thankful to VIT-CHENNAI for providing us with resources and fully equipped laboratory for carrying out the experiments. We are also thankful to the mentor, guide, co- author Dr. Jeyanthi Subramaniam and her research scholar Mr. Yuvraj for helping and providing us with basics and knowledge for the study of the results and its development.

\section{REFERENCES}

1. Mordiguine, M. Interference Control in Computer and Microprocessors Based Equipment's; Don White Consultant Inc.: Virginia, 1984.

2. Violette, J. L. N.; White, D. R. J.; Violette, M. F. Electromagnetic Compatibility Handbook; Van Nostrand Reinhold Company: New York, 1987.

3. F. Moglie, D. Micheli, S. Laurenzi, M. Marchetti, V.M. Primiani, Carbon 50 (2012) 1972-1980.

4. M. Jalali, S. Dauterstedt, A. Michaud, R. Wuthrich, Compos. Part B-Eng. 42 (2011) 1420-1426.

5. W. Jou, H. Cheng, C. Hsu, J. Alloys Comp. 434435 (2007) 641-645.

6. S. Mathew, P.R. Rejikumar, J. Yohannan, K.T. Mathew, N.V. Unnikrishnan, J. Alloys Comp. 462 (2008) 456-459. 